Article

\title{
Inspection Strategy under Indeterminacy Based on Neutrosophic Coefficient of Variation
}

\author{
Muhammad Aslam * (D) and Mansour Sattam Aldosari \\ Department of Statistics, Faculty of Science, King Abdulaziz University, Jeddah 21589, Saudi Arabia; \\ msattam@hotmail.com \\ * Correspondence: magmuhammad@kau.edu.sa or aslam_ravian@hotmail.com; Tel.: +966-593-329-841
}

Received: 4 January 2019; Accepted: 30 January 2019; Published: 9 February 2019

check for updates

\begin{abstract}
The existing sampling plans which use the coefficient of variation (CV) are designed under classical statistics. These available sampling plans cannot be used for sentencing if the sample or the population has indeterminate, imprecise, unknown, incomplete or uncertain data. In this paper, we introduce the neutrosophic coefficient of variation (NCV) first. We design a sampling plan based on the NCV. The neutrosophic operating characteristic (NOC) function is then given and used to determine the neutrosophic plan parameters under some constraints. The neutrosophic plan parameters such as neutrosophic sample size and neutrosophic acceptance number are determined through the neutrosophic optimization solution. We compare the efficiency of the proposed plan under the neutrosophic statistical interval method with the sampling plan under classical statistics. A real example which has indeterminate data is given to illustrate the proposed plan.
\end{abstract}

Keywords: neutrosophic statistical interval method; classical statistics; fuzzy logic; producer's risk; consumer's risk

\section{Introduction}

The sampling plan is an important tool for statistical quality control (SQC) which is used for the inspection of finished product batches before they are sent to the market [1]. Cheap inspections, which require less effort and minimized risks, are the main targets during the inspection process. These targets can be only achieved by applying a well-designed sampling plan. The decision regarding the product lot is made based on sample information. Ineffective sampling plans may mislead the experimenter, causing them to reject a good lot (producer's risk) and accepting a bad lot (consumer's risk). The supplier wishes to protect lots produced at permissible standards and the customer wants to reduce the chance of accepting a bad lot of the product. A well-designed sampling plan is based on the optimal plan parameters where the sample size required for the inspection and risks involved are at a minimum level.

Two major types of sampling plans are the attribute sampling plan and variable sampling plan. Variable sampling plans are designed for when the quality characteristic is measurable and attribute sampling plans are designed for a quality characteristic expressed as 'go, no-go'. The attribute sampling plans are easy to apply in practice but yield less information than variable sampling. The variable sampling plans can be applied to inspection lots using smaller sample sizes, while attribute plans require larger sample sizes to attain the same protection for the supplier and customer. Based on the advantages of the variable sampling over the attribute sampling plans, several authors from a variety of fields worked on designing variable sampling plans. For example, the authors of Reference [2] introduced the plan for the coefficient of variation $(\mathrm{CV})$, and they proposed a CV-based plan for two-stage sampling. In addition, the authors of Reference [3] presented the design of a CV-based plan using repetitive sampling. 
According to Yan et al. [4], the relative measure is called the coefficient of variation (CV). This is the ratio of standard deviation (SD), meaning it has been widely used to measure the relative variation to its mean in a variety of fields. For example, References [5-8] applied the CV in the testing of material reliability and the average quality characteristic used to measure the product quality. According to Reference [9], "in certain scenarios, the practitioner is not interested in the changes in the mean or the standard deviation but is instead interested in the relative variability compared with the mean". Due to the wide applications of the $\mathrm{CV}$, several authors have designed sampling plans, this includes a proposed multiple dependent state sampling plan for the CV [4], a proposed CV-based plan for two stage sampling [2], and presenting the designing of CV based planning using the repetitive sampling [3].

The sampling plans in the literature are applied for the inspection of the submitted lot of products under the assumption that all the observations are determined. The fuzzy approach is applied when there is uncertainty in the proportion parameters. The sampling plans which use the fuzzy approach can be applied for the inspection of a product lots with ambiguous proportion parameters. In recent years, several authors contributed excellent work on the design of sampling plan using the fuzzy approach, the authors of Reference [10] proposed fuzzy attribute plans for multiple dependent state sampling, the authors of Reference [11] designed the fuzzy plan using double sampling, the authors of Reference [12] proposed the fuzzy sampling plan using the Poisson distribution, the authors of Reference [13] studied the fuzzy operating characteristics curve, the authors of References $[14,15]$ proposed the double and sequential sampling plan using the fuzzy logic, and the authors of Reference [16] proposed the fuzzy plan using gamma distribution. More details can be seen in Reference [17].

Neutrosophic logic is the generalization of fuzzy logic as introduced by Reference [18]. Neutrosophic logic consists of measures of the truth, falsehood, and indeterminacy. Smarandache [19] introduced neutrosophic statistics using neutrosophic numbers. Neutrosophic statistics are the generalization of classical statistics, which can be applied when the data is indeterminate. This means that neutrosophic statistics can be applied to analyze data which may have some uncertain or unclear observations. The neutrosophic analysis provides the output in an indeterminacy interval rather than the determined values. Therefore, it is effective when applied under uncertain conditions. Recently, the authors of References $[20,21]$ introduced the neutrosophic statistical interval method for the rock measuring problem. According to Reference [22], "observations include human judgments, and evaluations and decisions, a continuous random variable of a production process should include the variability caused by human subjectivity or measurement devices, or environmental conditions. These variabilities can create vagueness in the measurement system." In this situation, when there is indeterminacy in the data, the lot sentencing cannot be done using the traditional sampling plans. To handle this situation, the authors of Reference [23] introduced the neutrosophic statistics in the area of acceptance sampling plans, as well as proposed sampling plans based on the process loss function under the neutrosophic statistical interval method, and the authors of Reference [24] designed the neutrosophic sampling plan using sudden death testing. Aslam and Raza's [25] design proposed a neutrosophic sampling plan for multiple manufacturing lines, the authors of Reference [26] proposed a plan for exponential distribution under neutrosophic statistics, and the authors of Reference [27] proposed a neutrosophic plan for using the regression estimator.

By exploring the literature and to the best of our knowledge, there is no work on the sampling plan for the $\mathrm{CV}$ using neutrosophic statistics. In this paper, we introduce the neutrosophic coefficient of variation (NCV). We first design a sampling plan based on the $\mathrm{CV}$ according to the neutrosophic statistical interval method. The neutrosophic operating characteristic (NOC) is given. We expect that the proposed sampling under the neutrosophic statistical interval method will be more effective and adequate than the sampling plan based on the classical statistics under the indeterminacy environment. 


\section{Neutrosophic Coefficient of Variation}

In this section, we first define the NCV and then design the sampling plan based on it.

Suppose that $X_{N i} \in\left\{X_{L}, X_{U}\right\}=i=1,2,3, \ldots, n_{N}$ is a neutrosophic random variable of size $n_{N}$ distributed as the neutrosophic normal distribution with neutrosophic mean $\mu_{N} \epsilon\left\{\mu_{L}, \mu_{U}\right\}$ and neutrosophic standard deviation $\sigma_{N} \in\left\{\sigma_{L}, \sigma_{U}\right\}$; for details, see [19]. The NCV is defined by

$$
C V_{N}=\sigma_{N} / \mu_{N} ; \mu_{N} \epsilon\left\{\mu_{L}, \mu_{U}\right\}, \sigma_{N} \epsilon\left\{\sigma_{L}, \sigma_{U}\right\}, C V_{N} \epsilon\left\{C V_{L}, C V_{U}\right\}
$$

It is important to note that $\mu_{N} \epsilon\left\{\mu_{L}, \mu_{U}\right\}$ and $\sigma_{N} \epsilon\left\{\sigma_{L}, \sigma_{U}\right\}$ are usually unknown in practice and are estimated using sample information. The best linear unbiased estimates (BLUE) of $\mu_{N} \epsilon\left\{\mu_{L}, \mu_{U}\right\}$ and $\sigma_{N} \epsilon\left\{\sigma_{L}, \sigma_{U}\right\}$ are $\bar{X}_{N} \epsilon\left\{\bar{X}_{L}, \bar{X}_{U}\right\}=\left\{\sum_{i=1}^{n} x_{i}^{L} / n_{L}, \sum_{i=1}^{n} x_{i}^{U} / n_{U}\right\}$ and $s_{N}=\left\{s_{L}, s_{U}\right\}=$ $\left\{\sqrt{\sum_{i=1}^{n}\left(x_{i}^{L}-\bar{X}_{L}\right)^{2} / n_{L}} \sqrt{\sum_{i=1}^{n}\left(x_{i}^{U}-\bar{X}_{U}\right)^{2} / n_{U}}\right\}$, respectively. The natural estimate of NCV is defined by

$$
\widehat{C V}_{N}=\frac{s_{N}}{\bar{X}_{N}} ; \bar{X}_{N} \epsilon\left\{\bar{X}_{L}, \bar{X}_{U}\right\}, s_{N}=\left\{s_{L}, s_{U}\right\}, C V_{N} \epsilon\left\{C V_{L}, C V_{U}\right\} .
$$

By following Reference [28], the neutrosophic statistic $\sqrt{X_{N}} / \widehat{C V}_{N} ; X_{N i} \epsilon\left\{X_{L}, X_{U}\right\}, \widehat{C V}_{N} \epsilon\left\{\widehat{C V}_{L}, \widehat{C V}_{U}\right\}$ follows the neutrosophic non-central chi-square distribution with a neutrosophic degree of freedom $n_{N}-1$ and neutrosophic non-centrality parameters $\delta_{N}=\sqrt{n_{N}} / \widehat{C V}_{N}$. The neutrosophic chi-square distribution is used to test the association between the qualitative data and having some uncertain observations. The neutrosophic chi-square distribution is reduced to chi-square distribution under classical statistics if all observations in the data are precise. Some more details on the neutrosophic distributions can be read in [19,24].

The sampling plans based on the NCV are stated as follows:

Step-1: Select a random sample of size $n_{N} \epsilon\left\{n_{L}, n_{U}\right\}$ and compute $\widehat{C V}_{N} \epsilon\left\{\widehat{C V}_{L}, \widehat{C V}_{U}\right\}$.

Step-2: Accept a lot of product if $\widehat{C V}_{N} \leq k_{N}$, otherwise reject a lot of the product.

Note that $k_{N} \epsilon\left\{k_{a L}, k_{a U}\right\}$ is a neutrosophic acceptance number.

The sampling plan based on the NCV has two neutrosophic plan parameters, $n_{N} \in\left\{n_{L}, n_{U}\right\}$ and $k_{N} \epsilon\left\{k_{a L}, k_{a U}\right\}$, that will be determined through the neutrosophic optimization solution. The proposed plan is a progression of the sampling plan based on CV under classical statistics reported in [29]. The proposed sampling plan is reduced to the aforementioned sampling plan [29] when there is no indeterminacy in the data.

The neutrosophic operating characteristic (NOC) function is derived as follows:

$$
\begin{gathered}
P_{N a}=P\left\{\widehat{C V}_{N} \leq k_{N}\right\} ; \widehat{C V}_{N} \epsilon\left\{\widehat{C V}_{L}, \widehat{C V}_{U}\right\}, k_{N} \epsilon\left\{k_{a L}, k_{a U}\right\} \\
P_{N a}=P\left\{\widehat{C V}_{N} \leq k_{N}\right\}=\mathrm{P}\left(\mathrm{t}_{\left.n_{N}-1, \sqrt{n_{N}} / C V_{N}>\sqrt{n_{N}} / k_{N}\right)}\right) \widehat{C V}_{N} \epsilon\left\{\widehat{C V}_{L}, \widehat{C V}_{U}\right\}, k_{N} \epsilon\left\{k_{a L}, k_{a U}\right\}, \\
n_{N} \epsilon\left\{n_{L}, n_{U}\right\} .
\end{gathered}
$$

Suppose that $\alpha$ and $\beta$ are the producer' risk and consumer's risk, respectively. To meet the producer and consumer requirements, the NOC should pass through $\left(p_{1}, 1-\alpha\right)$ and $\left(p_{2}, \beta\right)$, where $p_{1}$ and $p_{2}$ are an acceptable quality level (AQL) and limiting quality level (LQL), respectively. The neutrosophic plan parameters of the proposed plan are determined through following neutrosophic optimization solution (NOS):

$$
\operatorname{minimize} n_{N} \epsilon\left\{n_{L}, n_{U}\right\}
$$

subject to

$$
P_{N a}\left(p_{1}=\widehat{C V}_{N}\right)=\mathrm{P}\left(\mathrm{t}_{n_{N}-1, \sqrt{n_{N}} / \mathrm{CV}_{\mathrm{AQL}}}>\sqrt{\mathrm{n}}_{\mathrm{N}} / k_{N}\right) \geq 1-\alpha ; k_{N} \epsilon\left\{k_{a L}, k_{a U}\right\} ; n_{N} \epsilon\left\{n_{L}, n_{U}\right\}
$$


and

$$
P_{N a}\left(p_{2}=\widehat{C V}_{N}\right)=\mathrm{P}\left(\mathrm{t}_{n_{N}-1, \sqrt{n_{N}} / \mathrm{CV}_{\mathrm{LQL}}}>\sqrt{\mathrm{n}_{\mathrm{N}}} / k_{N}\right) \leq \beta ; k_{N} \epsilon\left\{k_{a L}, k_{a U}\right\} ; n_{N} \epsilon\left\{n_{L}, n_{U}\right\} .
$$

The plan parameters $n_{N} \epsilon\left\{n_{L}, n_{U}\right\}$ and $k_{N} \epsilon\left\{k_{a L}, k_{a U}\right\}$ are determined through the above NOS using the search grid method. It is noted that several combinations of $n_{N} \in\left\{n_{L}, n_{U}\right\}$ and $k_{N} \in\left\{k_{a L}, k_{a U}\right\}$ met the given conditions. The plan parameters $n_{N} \epsilon\left\{n_{L}, n_{U}\right\}$ and $k_{N} \epsilon\left\{k_{a L}, k_{a U}\right\}$ are selected at smaller values of $n_{N} \in\left\{n_{L}, n_{U}\right\}$. We used the following algorithm to find the neutrosophic plan parameters. The codes to make the tables are available from the authors upon request.

Step-1: Pre-fix the values of $\alpha, \beta, \mathrm{AQL}$, and LQL.

Step-2: Define a range for $n_{N}$, say $2<n_{N}<1000$. Determine the probabilities using Equations (6) and (7).

Step-3: Using search grid method, record those combinations from 10,000 combinations of plan parameters where $n_{N} \epsilon\left\{n_{L}, n_{U}\right\}$ is minimum.

Note here that the proposed NOS approaches the optimization solution under classical statistics if $k_{a L}=k_{a U}$ and $n_{L}=n_{U}$. Table 1 shows that plan parameters $n_{N} \in\left\{n_{L}, n_{U}\right\}$ and $k_{N} \epsilon\left\{k_{a L}, k_{a U}\right\}$ when $\alpha=5 \%$ and $\beta=10 \%$. Table 2 shows that plan parameters $n_{N} \epsilon\left\{n_{L}, n_{U}\right\}$ and $k_{N} \epsilon\left\{k_{a L}, k_{a U}\right\}$ when $\alpha=5 \%$ and $\beta=5 \%$. We note from Tables 1 and 2 that when the AQL is fixed, $n_{N} \in\left\{n_{L}, n_{U}\right\}$ decreases while $k_{N} \epsilon\left\{k_{a L}, k_{a U}\right\}$ increases. Further, for the fixed values of $\alpha, n_{N} \epsilon\left\{n_{L}, n_{U}\right\}$ decreases as $\beta$ increses.

Table 1. The neutrosophic fuzzy plan parameters when $\alpha=0.05, \beta=0.10$.

\begin{tabular}{cccccc}
\hline$C V_{A Q L}$ & $C V_{L Q L}$ & $n_{N} \epsilon\left\{n_{L}, n_{U}\right\}$ & $k_{N} \epsilon\left\{\boldsymbol{k}_{a L}, \boldsymbol{k}_{a U}\right\}$ & $\boldsymbol{P}_{N a}\left(p_{1}\right)$ & $\boldsymbol{P}_{N a}\left(p_{2}\right)$ \\
\hline 0.05 & 0.06 & $\{161,265\}$ & $\{0.05546,0.05637\}$ & $\{0.9759,0.9983\}$ & $\{0.0933,0.0861\}$ \\
& 0.07 & $\{61,167\}$ & $\{0.06092,0.06456\}$ & $\{0.9924,0.9999\}$ & $\{0.0854,0.08353\}$ \\
& 0.08 & $\{37,92\}$ & $\{0.06506,0.06616\}$ & $\{0.9954,0.9999\}$ & $\{0.0642,0.0111\}$ \\
& 0.09 & $\{21,40\}$ & $\{0.06858,0.06953\}$ & $\{0.9922,0.9997\}$ & $\{0.0778,0.0260\}$ \\
0.06 & 0.10 & $\{43,58\}$ & $\{0.06923,0.07187\}$ & $\{0.9998,0.9999\}$ & $\{0.00296,0.00163\}$ \\
& 0.07 & $\{332,407\}$ & $\{0.06630,0.06641\}$ & $\{0.9966,0.9988\}$ & $\{0.0910,0.0752\}$ \\
& 0.08 & $\{100,116\}$ & $\{0.06766,0.07080\}$ & $\{0.9659,0.9970\}$ & $\{0.0168,0.0443\}$ \\
& 0.09 & $\{72,146\}$ & $\{0.07393,0.07485\}$ & $\{0.9974,0.9999\}$ & $\{0.0190,0.0024\}$ \\
& 0.10 & $\{23,72\}$ & $\{0.07674,0.07949\}$ & $\{0.9723,0.9999\}$ & $\{0.072,0.0085\}$ \\
0.07 & 0.11 & $\{23,37\}$ & $\{0.07711,0.08012\}$ & $\{0.9747,0.9980\}$ & $\{0.0289,0.0128\}$ \\
& 0.08 & $\{323,412\}$ & $\{0.07564,0.07604\}$ & $\{0.9799,0.9934\}$ & $\{0.08757,0.0818\}$ \\
& 0.09 & $\{125,145\}$ & $\{0.07925,0.08196\}$ & $\{0.9821,0.9982\}$ & $\{0.0330,0.0698\}$ \\
& 0.10 & $\{120,150\}$ & $\{0.08340,0.08485\}$ & $\{0.9985,0.9998\}$ & $\{0.0060,0.0051\}$ \\
& 0.11 & $\{61,97\}$ & $\{0.09125,0.09208\}$ & $\{0.9996,0.9999\}$ & $\{0.0354,0.0138\}$ \\
0.08 & 0.12 & $\{21,90\}$ & $\{0.09236,0.10763\}$ & $\{0.9814,1.000\}$ & $\{0.0859,0.0930\}$ \\
& 0.09 & $\{748,928\}$ & $\{0.08431,0.08450\}$ & $\{0.9814,0.9961\}$ & $\{0.0079,0.0086\}$ \\
& 0.10 & $\{115,152\}$ & $\{0.08918,0.09061\}$ & $\{0.9601,0.9897\}$ & $\{0.0562,0.0559\}$ \\
& 0.11 & $\{68,80\}$ & $\{0.09145,0.09623\}$ & $\{0.9541,0.9949\}$ & $\{0.0291,0.0643\}$ \\
& 0.12 & $\{47,70\}$ & $\{0.1001,0.1042\}$ & $\{0.9926,0.9998\}$ & $\{0.0639,0.0677\}$ \\
& 0.13 & $\{33,60\}$ & $\{0.10309,0.11169\}$ & $\{0.9905,0.9999\}$ & $\{0.0573,0.0713\}$ \\
& 0.10 & $\{655,820\}$ & $\{0.09459,0.09559\}$ & $\{0.9672,0.9963\}$ & $\{0.0270,0.0553\}$ \\
& 0.11 & $\{126,181\}$ & $\{0.10066,0.10138\}$ & $\{0.9703,0.9918\}$ & $\{0.0971,0.0740\}$ \\
& 0.12 & $\{77,107\}$ & $\{0.10393,0.10514\}$ & $\{0.9731,0.9929\}$ & $\{0.0555,0.0401\}$ \\
& 0.13 & $\{62,85\}$ & $\{0.10558,0.11026\}$ & $\{0.9736,0.9983\}$ & $\{0.0223,0.0283\}$ \\
& 0.14 & $\{41,74\}$ & $\{0.10848,0.11624\}$ & $\{0.9694,0.9998\}$ & $\{0.0265,0.0237\}$ \\
\hline
\end{tabular}


Table 2. The neutrosophic fuzzy plan parameters when $\alpha=0.05, \beta=0.05$.

\begin{tabular}{cccccc}
\hline$C V_{A Q L}$ & $C V_{L Q L}$ & $n_{N} \epsilon\left\{n_{L}, n_{U}\right\}$ & $k_{N} \epsilon\left\{\boldsymbol{k}_{a L}, \boldsymbol{k}_{a U}\right\}$ & $\boldsymbol{P}_{N a}\left(\boldsymbol{p}_{1}\right)$ & $\boldsymbol{P}_{N a}\left(\boldsymbol{p}_{2}\right)$ \\
\hline 0.05 & 0.06 & $\{320,545\}$ & $\{0.05432,0.05689\}$ & $\{0.9859,0.9999\}$ & $\{0.0090,0.0456\}$ \\
& 0.07 & $\{81,143\}$ & $\{0.05806,0.06016\}$ & $\{0.9809,0.9997\}$ & $\{0.0403,0.0099\}$ \\
& 0.08 & $\{55,102\}$ & $\{0.06271,0.06716\}$ & $\{0.9963,0.9999\}$ & $\{0.0143,0.0126\}$ \\
& 0.09 & $\{24,85\}$ & $\{0.06492,0.07410\}$ & $\{0.9817,1.000\}$ & $\{0.0352,0.0126\}$ \\
0.06 & 0.10 & $\{18,65\}$ & $\{0.06857,0.07824\}$ & $\{0.9875,1.000\}$ & $\{0.0410,0.0081\}$ \\
& 0.07 & $\{289,490\}$ & $\{0.06473,0.06561\}$ & $\{0.9519,0.9986\}$ & $\{0.0003,0.0113\}$ \\
& 0.08 & $\{109,163\}$ & $\{0.07068,0.07222\}$ & $\{0.9958,0.9998\}$ & $\{0.0474,0.0434\}$ \\
& 0.09 & $\{51,137\}$ & $\{0.07101,0.07700\}$ & $\{0.9696,0.9999\}$ & $\{0.0202,0.0970\}$ \\
& 0.10 & $\{39,79\}$ & $\{0.07241,0.07259\}$ & $\{0.9679,0.9959\}$ & $\{0.0097,0.0004\}$ \\
0.07 & 0.11 & $\{23,76\}$ & $\{0.07624,0.07805\}$ & $\{0.9686,0.9999\}$ & $\{0.0256,0.0002\}$ \\
& 0.08 & $\{412,689\}$ & $\{0.07509,0.07618\}$ & $\{0.9818,0.9994\}$ & $\{0.0418,0.0401\}$ \\
& 0.09 & $\{110,277\}$ & $\{0.07967,0.08018\}$ & $\{0.9803,0.9996\}$ & $\{0.0494,0.0058\}$ \\
& 0.10 & $\{50,104\}$ & $\{0.08274,0.08277\}$ & $\{0.9671,0.9958\}$ & $\{0.0498,0.008\}$ \\
& 0.11 & $\{41,102\}$ & $\{0.08300,0.09190\}$ & $\{0.9560,0.9999\}$ & $\{0.0168,0.0112\}$ \\
0.08 & 0.12 & $\{28,118\}$ & $\{0.09056,0.10651\}$ & $\{0.9864,1.000\}$ & $\{0.0426,0.0476\}$ \\
& 0.09 & $\{431,789\}$ & $\{0.08458,0.08571\}$ & $\{0.9538,0.9976\}$ & $\{0.0411,0.0311\}$ \\
& 0.10 & $\{179,204\}$ & $\{0.08845,0.08982\}$ & $\{0.9775,0.9934\}$ & $\{0.0163,0.0222\}$ \\
& 0.11 & $\{80,177\}$ & $\{0.09264,0.09787\}$ & $\{0.9778,0.9999\}$ & $\{0.0269,0.0215\}$ \\
& 0.12 & $\{54,91\}$ & $\{0.09739,0.09822\}$ & $\{0.9882,0.9989\}$ & $\{0.0305,0.0088\}$ \\
& 0.13 & $\{35,106\}$ & $\{0.09947,0.10929\}$ & $\{0.9797,0.9999\}$ & $\{0.0315,0.0123\}$ \\
0.09 & 0.10 & $\{673,906\}$ & $\{0.09487,0.09513\}$ & $\{0.9762,0.9921\}$ & $\{0.0322,0.0206\}$ \\
& 0.11 & $\{154,229\}$ & $\{0.09869,0.09937\}$ & $\{0.9555,0.9869\}$ & $\{0.0398,0.0217\}$ \\
& 0.12 & $\{93,167\}$ & $\{0.10448,0.10806\}$ & $\{0.9859,0.9998\}$ & $\{0.0446,0.0388\}$ \\
& 0.13 & $\{69,125\}$ & $\{0.10876,0.11385\}$ & $\{0.9928,0.9999\}$ & $\{0.0328,0.0287\}$ \\
& 0.14 & $\{41,111\}$ & $\{0.10638,0.12125\}$ & $\{0.9521,0.9999\}$ & $\{0.0193,0.0271\}$ \\
\hline
\end{tabular}

\section{Advantage of the Proposed Plan}

In this section, we discuss the advantage of the proposed plan under the neutrosophic interval method over the plan designed under classical statistics. The neutrosophic sample size has the form $n_{N}=n+u I$, where $n$ is the determined part and also the parameter of the plan under classical statistics,

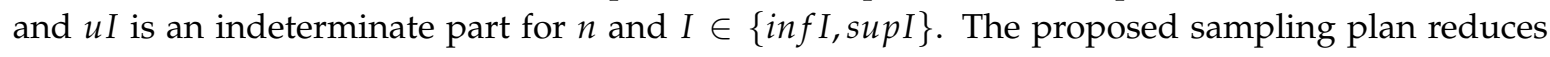
the plan under classical statistics when $n_{L}=n_{U}=n$. According to References [20,21], a method which provides the parameters in range/interval is considered more effective than the method which provides the determined value under the uncertainty environment. The values of $n_{N} \epsilon\left\{n_{L}, n_{U}\right\}$ and $n$ are reported in Table 3 when $\alpha=0.05$ and $\beta=0.05$.

From Table 4, the proposed plan has the parameter in the indeterminacy interval while the existing sampling plan under classical statistics has determined values. For example, when AQL $=0.05$ and $\mathrm{LQL}=0.06$, the proposed plan has the neutrosophic interval in sample size $n_{N}=320+320 I$, $I \in\{0,1.7031\}$. Then, the neutrosophic sample size is $n_{N} \epsilon\{320,545\}$ for $I \in\{0,1.7031\}$. So, under conditions of uncertainty, the experimenter should select a sample size between 320 and 545 . The existing sampling plan provides the determined value of 320. This means that the proposed plan is more effective and adequate under uncertainty and this theory coincides with the findings of $[20,21]$. 
Table 3. The neutrosophic fuzzy plan parameters when $\alpha=0.05, \beta=0.05$.

\begin{tabular}{cccc}
\hline$C V_{A Q L}$ & $C V_{L Q L}$ & $n_{N} \epsilon\left\{n_{L}, n_{U}\right\}$ & $n$ \\
\hline 0.05 & 0.06 & $\{320,545\}$ & 320 \\
& 0.07 & $\{81,143\}$ & 81 \\
& 0.08 & $\{55,102\}$ & 55 \\
0.06 & 0.09 & $\{24,85\}$ & 24 \\
& 0.10 & $\{18,65\}$ & 18 \\
& 0.07 & $\{289,490\}$ & 289 \\
& 0.08 & $\{109,163\}$ & 109 \\
0.07 & 0.09 & $\{51,137\}$ & 51 \\
& 0.10 & $\{39,79\}$ & 39 \\
& 0.11 & $\{23,76\}$ & 23 \\
& 0.08 & $\{412,689\}$ & 412 \\
& 0.09 & $\{110,277\}$ & 110 \\
0.08 & 0.10 & $\{50,104\}$ & 50 \\
& 0.11 & $\{41,102\}$ & 41 \\
& 0.12 & $\{28,118\}$ & 28 \\
& 0.09 & $\{431,789\}$ & 431 \\
& 0.10 & $\{179,204\}$ & 179 \\
& 0.11 & $\{80,177\}$ & 80 \\
0.09 & 0.12 & $\{54,91\}$ & 54 \\
& 0.13 & $\{35,106\}$ & 35 \\
& 0.10 & $\{673,906\}$ & 673 \\
& 0.11 & $\{154,229\}$ & 154 \\
& 0.12 & $\{93,167\}$ & 93 \\
& 0.13 & $\{69,125\}$ & 69 \\
& 0.14 & $\{41,111\}$ & 41 \\
\hline \multirow{3}{*}{0.14} & &
\end{tabular}

\section{Case Study}

In this section, we discuss the application of the proposed sampling plan using real data from the concrete industry. Concrete is an important material in building and road construction. The comprehensive strength is the maximum load which the material can sustain without any fracture. This quality characteristic is measured for engineering buildings and other structures. Therefore, engineers are more concerned about the stability of concrete strength. The comprehensive strength of concrete is not always a crisp value in practice. According to Reference [30] "Input fields of the fuzzy expert system are the weight percent of cement, water, blast furnace slag, fly ash, super plasticizer, fine aggregate, coarse aggregate, and age of the concrete". Measuring the strength of concrete is a complex system and there is a chance of having some indeterminate, incomplete, imprecise, or vague (unclear) observations. In this situation, the presentation of data in a set is called neutrosophication, making the proposed plan more effective in application than the existing sampling plan. The existing plan can be applied if data are deneutrosophied by replacing the neutrosophic data with the mid values of data, see [19]. Imprecise observations lead to uncertainty in the plan parameters needed for the inspection of a concrete product. Suppose that for this lot sentencing, $\mathrm{AQL}=0.05, \mathrm{LQL}=0.09, \alpha=5 \%$, and $\beta=10 \%$. The neutrosophic plan parameters from Table 1 are $n_{N} \epsilon\{21,40\}$ and $k_{N} \epsilon\{0.0685,0.0695\}$. The comprehensive strength of 25 concrete mixture specimens having some indeterminate, incomplete, imprecise, and vague observations is shown in Table 4.

Table 4. The data of concrete mixture specimens.

\begin{tabular}{lllll}
\hline Column 1 & Column 2 & Column 3 & Column 4 & Column 5 \\
\hline$(36.3,36.9)$ & $(40.1,40.1)$ & $(31.8,32.1)$ & $(33.6,33.6)$ & $(34.9,35.2)$ \\
$(31.2,31.2)$ & $(32.8,32.8)$ & $(25.8,25.8)$ & $(30.8,32.2)$ & $(32.9,32.9)$ \\
$(30.9,30.9)$ & $(31.9,32.4)$ & $(35.6,35.6)$ & $(30.9,30.9)$ & $(27.8,29.1)$ \\
$(24.9,24.9)$ & $(31.6,31.6)$ & $(27.9,28.2)$ & $(33.7,33.7)$ & $(38.4,38.4)$ \\
$(28.5,28.9)$ & $(31.4,31.8)$ & $(26.9,26.9)$ & $(32.7,32.7)$ & $(34.1,34.6)$ \\
\hline
\end{tabular}


The NCV for the real data is computed as follows:

$\bar{X}_{N} \epsilon\{31.89,32.13\}$ and $s_{N} \epsilon\{3.64,3.67\}$.

So,

$\widehat{C V}_{N} \epsilon\{0.1133,0.1152\}, \bar{X}_{N} \epsilon\{31.89,32.13\}$, and $s_{N} \epsilon\{3.64,3.67\}$.

The proposed plan is implemented as follows:

Step-1: Select a random sample of size $n_{N} \epsilon\{21,40\}$ and compute $\widehat{C V}_{N} \in\{0.1133,0.1152\}$.

Step-2: Reject a lot of concrete product since $\widehat{C V}_{N} \in\{0.1133,0.1152\}>k_{N} \epsilon\{0.0685,0.0695\}$.

\section{Concluding Remarks}

In this manuscript, we originally designed a sampling plan based on the neutrosophic coefficient of variation $(\mathrm{NCV})$ which can be applied when observations/parameters are indeterminate in practice. The structure of the proposed plan is given to find the neutrosophic plan parameters. The application of the proposed neutrosophic plan is given with the aid of company data. The proposed plan under the neutrosophic interval method is more effective and adequate under the uncertainty environment. The proposed plan using big data from the industry can be studied as future research. The estimation and properties of neutrosophic normal distribution and neutrosophic chi-square distribution can be considered as future research.

Author Contributions: Conceived and designed the experiments, M.A.; Performed the experiments, M.A.; Analyzed the data, M.A. and M.S.A.; Contributed reagents/materials/analysis tools, M.A.; Wrote the paper, M.A. and M.S.A.

Funding: This article was funded by the Deanship of Scientific Research (DSR) at King Abdulaziz University, Jeddah. The authors, therefore, acknowledge with thanks DSR technical and financial support.

Acknowledgments: The authors are deeply thankful to editors and reviewers for their valuable suggestions to improve the quality of this manuscript.

Conflicts of Interest: The authors declare no conflict of interest regarding this paper.

\section{References}

1. Montgomery, D.C. Introduction to Statistical Quality Control; John Wiley \& Sons: Hoboken, NJ, USA, 2007.

2. Yan, A.; Liu, S.; Dong, X. Variables two stage sampling plans based on the coefficient of variation. J. Adv. Mech. Des. Syst. Manuf. 2016, 10, JAMDSM0002. [CrossRef]

3. Yan, A.-J.; Aslam, M.; Azam, M.; Jun, C.-H. Developing a variable repetitive group sampling plan based on the coefficient of variation. J. Ind. Prod. Eng. 2017, 34, 398-405. [CrossRef]

4. Yan, A.; Liu, S.; Dong, X. Designing a multiple dependent state sampling plan based on the coefficient of variation. SpringerPlus 2016, 5, 1447. [CrossRef] [PubMed]

5. Reed, G.F.; Lynn, F.; Meade, B.D. Use of coefficient of variation in assessing variability of quantitative assays. Clin. Diagn. Lab. Immunol. 2002, 9, 1235-1239. [CrossRef] [PubMed]

6. Pereira, M.A.; Weggemans, R.M.; Jacobs, D.R., Jr.; Hannan, P.J.; Zock, P.L.; Ordovas, J.M.; Katan, M.B. Within-person variation in serum lipids: Implications for clinical trials. Int. J. Epidemiol. 2004, 33, 534-541. [CrossRef] [PubMed]

7. Parsons, H.M.; Ekman, D.R.; Collette, T.W.; Viant, M.R. Spectral relative standard deviation: A practical benchmark in metabolomics. Analyst 2009, 134, 478-485. [CrossRef] [PubMed]

8. He, X.; Oyadiji, S. Application of coefficient of variation in reliability-based mechanical design and manufacture. J. Mater. Process. Technol. 2001, 119, 374-378. [CrossRef]

9. Yeong, W.C.; Khoo, M.B.C.; Teoh, W.L.; Castagliola, P. A control chart for the multivariate coefficient of variation. Qual. Reliab. Eng. Int. 2016, 32, 1213-1225. [CrossRef]

10. Afshari, R.; Sadeghpour Gildeh, B. Designing a multiple deferred state attribute sampling plan in a fuzzy environment. Am. J. Math. Manag. Sci. 2017, 36, 328-345. [CrossRef]

11. Sadeghpour Gildeh, B.; Baloui Jamkhaneh, E.; Yari, G. Acceptance single sampling plan with fuzzy parameter. Iran. J. Fuzzy Syst. 2011, 8, 47-55. 
12. Divya, P. Quality interval acceptance single sampling plan with fuzzy parameter using poisson distribution. Int. J. Adv. Res Technol. 2012, 1, 115-125.

13. Turanoğlu, E.; Kaya, İ.; Kahraman, C. Fuzzy acceptance sampling and characteristic curves. Int. J. Comput. Intell. Syst. 2012, 5, 13-29. [CrossRef]

14. Jamkhaneh, E.B.; Gildeh, B.S. Acceptance double sampling plan using fuzzy poisson distribution. World Appl. Sci. J. 2011, 15, 1692-1702.

15. Jamkhaneh, E.B.; Gildeh, B.S. Sequential sampling plan using fuzzy sprt. J. Intell. Fuzzy Syst. 2013, 25, 785-791.

16. Venkateh, A.; Elango, S. Acceptance sampling for the influence of trh using crisp and fuzzy gamma distribution. Aryabhatta J. Math. Inform. 2014, 6, 119-124.

17. Elango, S.; Venkatesh, A.; Sivakumar, G. A fuzzy mathematical analysis for the effect of trh using acceptance sampling plans. Int. J. Pure Appl. Math. 2017, 117, 1-11.

18. Smarandache, F. Neutrosophic logic-A generalization of the intuitionistic fuzzy logic. In Multispace $\mathcal{E}$ Multistructure. Neutrosophic Transdisciplinarity (100 Collected Papers of Science); Infinite Study: El Segundo, CA, USA, 2010; Volume 4, p. 396.

19. Smarandache, F. Introduction to Neutrosophic Statistics; Infinite Study: El Segundo, CA, USA, 2014.

20. Chen, J.; Ye, J.; Du, S. Scale effect and anisotropy analyzed for neutrosophic numbers of rock joint roughness coefficient based on neutrosophic statistics. Symmetry 2017, 9, 208. [CrossRef]

21. Chen, J.; Ye, J.; Du, S.; Yong, R. Expressions of rock joint roughness coefficient using neutrosophic interval statistical numbers. Symmetry 2017, 9, 123. [CrossRef]

22. Senturk, S.; Erginel, N. Development of fuzzy $\mathrm{x}^{-} \sim-\mathrm{r} \sim$ and $\mathrm{x}^{-} \sim-\mathrm{s} \sim$ control charts using $\alpha$-cuts. Inf. Sci. 2009, 179, 1542-1551. [CrossRef]

23. Aslam, M. A new sampling plan using neutrosophic process loss consideration. Symmetry 2018, 10, 132. [CrossRef]

24. Aslam, M.; Arif, O. Testing of grouped product for the weibull distribution using neutrosophic statistics. Symmetry 2018, 10, 403. [CrossRef]

25. Aslam, M.; Raza, M.A. Design of new sampling plans for multiple manufacturing lines under uncertainty. Int. J. Fuzzy Syst. 2018, 1-15. [CrossRef]

26. Aslam, M. Design of sampling plan for exponential distribution under neutrosophic statistical interval method. IEEE Access 2018, 6, 64153-64158. [CrossRef]

27. Aslam, M.; AL-Marshadi, A. Design of sampling plan using regression estimator under indeterminacy. Symmetry 2018, 10, 754. [CrossRef]

28. Iglewicz, B.; Myers, R.H.; Howe, R.B. On the percentage points of the sample coefficient of variation. Biometrika 1968, 55, 580-581. [CrossRef]

29. Tong, Y.; Chen, Q. Sampling inspection by variables for coefficient of variation. Theor. Appl. Probab. 1991, 3, 315-327.

30. Abolpour, B.; Abolpour, B.; Abolpour, R.; Bakhshi, H. Estimation of concrete compressive strength by a fuzzy logic model. Res. Chem. Intermed. 2013, 39, 707-719. [CrossRef]

(C) 2019 by the authors. Licensee MDPI, Basel, Switzerland. This article is an open access article distributed under the terms and conditions of the Creative Commons Attribution (CC BY) license (http://creativecommons.org/licenses/by/4.0/). 